Article

\title{
On the Interaction of Information and Matter: The Case Study about the Language and Brain
}

\author{
Liujun Chen, Yan Liu*, Jiawei Chen, Xiaomeng Li and Zengru Di \\ School of Systems Science, Beijing Normal University, Beijing 100875, China; chenlj@bnu.edu.cn (L.C.); \\ chenjiawei@bnu.edu.cn (J.C.); lixiaomeng@bnu.edu.cn (X.L.); zdi@bnu.edu.cn (Z.D.) \\ * Correspondence: bnuliuyan@bnu.edu.cn
}

\begin{abstract}
Since matter, energy and information are the three major components of the world, is there an interaction between information and matter? In the present work, the coevolution of human language and brain is taken as a case of interaction between information and brain. Some evidence that may show interactions between human language and brain revealed by previous researches is summarized in this paper, such as the language areas in the cerebral cortex of the modern human brain, the evolution of human language and brain in human history, and the genetic basis for the evolution of language. Based on the evidence, a dynamic model is developed to investigate the possible mechanism of coevolution of human language and brain. In the model, human language development and brain development reinforce each other: the developmental level of language can be promoted by advances in brain function due to language-related gene mutations, in turn, whether such mutations are selected positively can be influenced by the current developmental level of language. The coevolution of human language and brain can be taken as a case of interaction between information and matter.
\end{abstract}

Keywords: information; matter; language; brain; evolution

\section{Introduction}

Matter, energy and information are the three major components of the world. In 1905, Einstein proposed the mass-energy equation $\mathrm{E}=\mathrm{MC}^{2}$, which clarified the interaction between matter and energy. Now, we have entered the information age. Since there is an interaction between energy and matter, is there an interaction between information and matter?

Information was first used as a scientific term by R.V. Hartley in his script "The Transmission of Information" in 1928. C. E. Shannon proposed the information theory in 1948, which gave a definition of information from a mathematical point of view [1]. A key concept in the information theory is Entropy. Entropy quantifies the amount of uncertainty involved in the value of a random variable or the outcome of a random process. Further, more concepts were derived from Entropy, such as Joint Entropy and Mutual Information, which describe the transfer of information by the method of mathematical statistics. Although information can be encoded into various forms for transmission and interpretation, it is always conveyed as the content of a message. N. Wiener, the founder of Cybernetics, said that "Information is a name for the content of what is exchanged with the outer world as we adjust to it, and make our adjustment felt upon it" [2].

Although the research on information has been going on for a century, the researches on the interaction between information and matter are rare. In fact, there are some cases that reveal the interaction between information and matter. One case is that ribosomes translate mRNA into protein. The mRNA carries genetic information encoded as a DNA sequence and the ribosomes translate this code to a specific sequence of amino acids and then synthesize proteins. This process reflects the interaction between information and matter. Another case is that a pheromone triggers a social response in members of the same species [3]. Pheromones act like hormones outside the body of the secreting individual and then impact the behavior of the receiving individuals. Although pheromones have properties of matter, they can cause changes in individuals and their behaviors by 
means of information transmission between individuals of the same species. Therefore, this case also can be regarded as a kind of interaction between information and matter. Then, are there other cases that reflect the interaction between information and matter?

Language is the main medium that humans use to exchange messages and communicate. Therefore, the essence of language is information. Due to different points of view and research methods, the definitions of "language" are diverse. Below are some of the definitions. Language is (1) audible, articulate, meaningful sound as produced by the action of the vocal organs; (2) systematic means of communicating ideas or feelings by using conventionalized signs, sounds, gestures, or marks with understood meanings; (3) the method of human communication, either spoken or written, consisting of the use of words in a structured and conventional way. The first two definitions of language are not human-specific since primates and other animals can also have this kind of language. The third definition is unique to the human language, which is the definition of language referred to in this paper.

There are several language areas in the cerebral cortex of the human brain, including Broca's area, Wernicke's area, angular gyrus and insular cortex [4]. All these language-related areas indicate a close relationship between human language and brain. From the perspective of human history, there must be coevolution of human language and brain. In this paper, some evidence that may show interactions between human language and brain is given. Based on the evidence, a dynamic model is developed to investigate the possible mechanism of coevolution of human language and brain, which can be used as another start point to explore interactions between information and matter.

\section{Evidence of Interaction between Human Language and Brain}

\subsection{Language Areas in Cerebral Cortex of Modern Human Brain}

Several areas in the cerebral cortex of modern human brain play a critical role in human language and speech ability, which can be taken as solid evidence of interaction between human language and brain.

Broca's area, located in the posterior section of the superior temporal gyrus (STG) in the (most commonly) left cerebral hemisphere, is associated with the production of language. It works in conjunction with working memory to allow a person to use verbal expression and spoken words. Damage to Broca's area can result in productive aphasia (also known as Broca's aphasia), or an inability to speak [5]. Wernicke's area is a critical language area in the posterior superior temporal lobe, connecting to Broca's area via a neural pathway. Wernicke's area is primarily involved in the comprehension or understanding of written and spoken language. Damage to this area results in receptive aphasia (also called Wernicke's aphasia) [6]. The angular gyrus, located mainly in the parietal lobe of the brain, is responsible for transferring visual information to Wernicke's area, in order to make meaning out of visually perceived words. It is also involved in a number of processes related to language, number processing, spatial cognition, memory retrieval, attention, and theory of mind [7]. The insular cortex, located underneath the outer lobes of the cerebral cortex, has direct connections to Broca's area and other language and speech centers. It may play an important role in coordinating higher-order cognitive aspects of language and speech production [8].

From the perspective of evolution, such correspondence between language ability and brain structure should be a result of their coevolution in human history. Some of the previous researches supporting such coevolution of human language and brain are discussed next.

\subsection{The Evolution of Human Language and Brain in Human History}

The questions of why and when human language emerged and how it evolved into its modern form are quite controversial since the origin of language began in the prehistory of mankind without any direct historical traces left. However, some meaningful inferences could be obtained from anatomical and archaeological researches on the human brain.

The two genera, Australopithecus and Homo, represent the lineages most importantly linked with human evolution. The genus Homo is generally considered to start from Homo habilis, who has 
evolved from one branch of Australopithecus 2 million years ago. The paleoneurological researches showed that roughly 1.8-2.0 million years ago, there is clear fossil evidence for a Homo lineage showing a more modern and enlarged Broca's area, expanded brain size. Moreover, cerebral asymmetries which correlate with right-handedness and left-right asymmetry of cognitive functioning was found to be strong and seemingly identical to those known for modern Homo sapiens $[9,10]$. Compared to that, the presence of Broca's area homologue in chimpanzees was also confirmed, but whether or not it exhibits humanlike asymmetry was not reliably determined due to its gross and microstructural variability in great apes [11].

The cranial capacity (brain size) is often used to characterize the development of the human brain (Figure 1). The cranial capacity of Homo habilis between 2.1 and 1.5 million years ago was about 510-690 ml, increasing by half compared to about 390-550 $\mathrm{ml}$ of Australopithecus afarensis, an extinct branch of Australopithecus. Homo erectus lived between 1.8 million and 200,000 years ago, whose cranial capacity was about $690-1200 \mathrm{ml}$. Homo heidelbergensis lived between 700,000 and 300,000 years ago, whose cranial capacity increased to about 880-1450 ml. Homo neanderthalensis lived between 250,000 and 40,000 years ago, whose cranial capacity was about 1170-1740 ml. Homo sapiens lived between 300,000 years and 10,000 years ago, whose cranial capacity was about 1090-1775 ml [12].

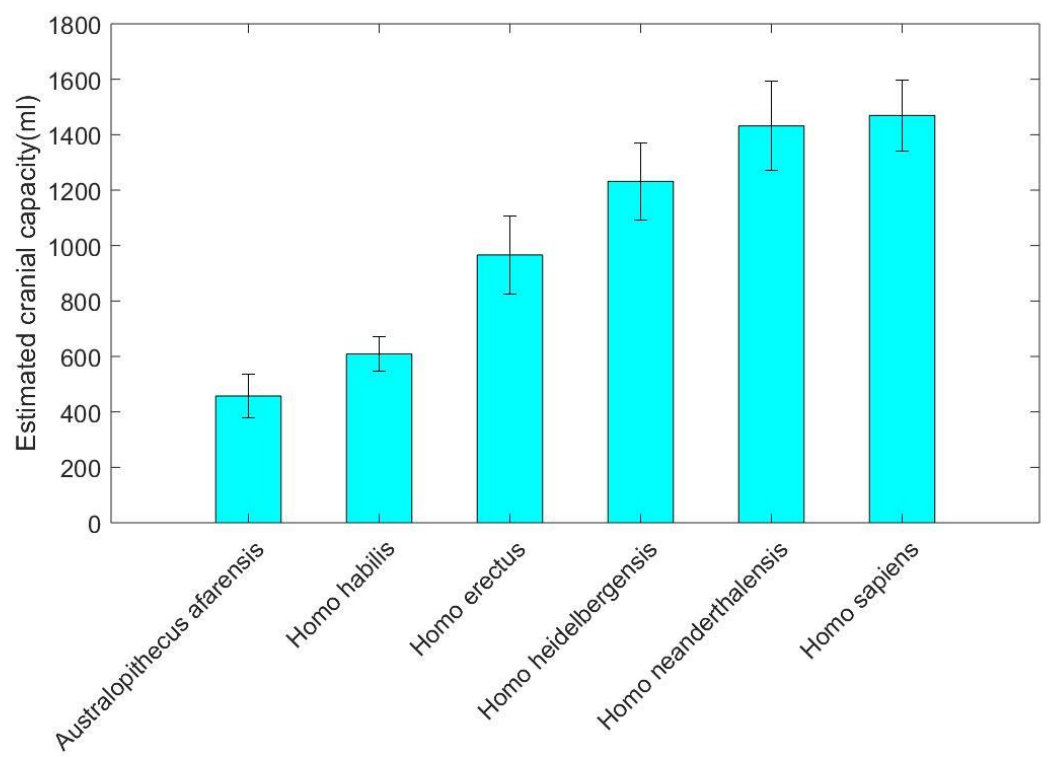

Figure 1. Evolution of human cranial capacity. Human cranial capacity experienced a rapid growth period from Homo habilis to Homo sapiens. Data is from reference [12].

From Figure 1 we can find that human brain size experienced a rapid growth period from Homo habilis to Homo sapiens. Some scientists pointed to the beginning of brain enlargement and the first appearance of stone tools in Homo habilis or early Homo erectus as a stimulus for language $[13,14]$. Leakey (1994) supposed that a protolanguage might have been produced by Homo habilis on the grounds that Broca's area and cerebral asymmetries in modern human brain were found on the skull of Homo habilis 2 million years ago [9,15].

However, developed brain function including the presence of language areas was just one aspect of physiological requirements for language ability, a larynx structure similar to modern humans is also essential. In fact, a fully descended vocal tract is required to emit vowels [i], [u] and [a], which are the base of modern human language. Until around 300,000 years ago, the curvature of the skull base of Archaic Homo sapiens reached the level of modern people so that the vocal tract could emit a full range of human speech, which met another key requirement for humans to acquire language skills[16]. Through analysis of the skull base and the lower jaw of Homo neanderthalensis, it is considered that the larynx is similar to the infants and other primates, so they were unlikely to speak like a modern human $[17,18]$. The complex verbal ability was considered the advantages of Homo sapiens over Homo neanderthalensis [19]. When modern humans entered Europe from 45,000 to 
42,000 years ago, they competed with Homo neanderthalensis there for resources until Homo neanderthalensis was extinct about 35,000 years ago.

For an overall view of human language evolution, Aitchison (2002) proposed a supposition of "language bonfire" which could be plotted on a graph as an S-shaped curve. According to this supposition, a simplified type of language began to emerge at least as early as 250,000 years ago and then developed slowly. At some time between 100,000 75,000 years ago, language reached a critical stage of sophistication and gained rapid development, which was compared to a process that ignition point arrived with a following massive blaze. By around 50,000 years ago, it was stabilizing into a steady state with the diffusion of culture from Africa to all over the world [20].

Although there is no universally accepted theory about the origin of language, researches indicated a close relationship between the development of human language and brain There is a possibility that the quick development of human language ability and rapid growth of brain size in human evolution may be mutually reinforced. On one hand, the development of human language facilitated social communication and improved production technologies which then stimulated brain development. On the other hand, the development of the human brain including the appearance of new encephalic regions are necessary as the physiological basis for speech and logical thinking. Such coevolution of human language and brain can be taken as an essential aspect of their interaction.

\subsection{Genetic Basis for the Possible Language-Brain Interaction}

More and more researches show that human language ability may be genetically transmitted. Studies have found the chromosomes and the genes associated with language and speech. Forkhead box protein P2 (FOXP2) is the first gene that was discovered to be related to language and speech ability. Scientists found that half the members of the KE family suffered from a language and speech disorder caused by a point mutation in the FOXP2 gene [21,22]. They found that the affected members of the KE family show significant underactivation relative to the unaffected members in Broca's area and speech related cortical and subcortical brain regions during the fMRI language experiments, which means that the FOXP2 gene is important in the development of the neural systems that related to language and speech [22]. Further researches introduced the two amino acid substitutions in human FOXP2 gene into the endogenous FOXP2 gene of mice $[23,24]$. The results showed that humanized FOXP2 allele impacts cortico-basal ganglia circuits, which might have been important for the evolution of language and speech [24]. The evolution of human FOXP2 gene might lead to differential tuning of corticostriatal systems and helped the human brain adapt for the acquisition of language and speech. Thus, the FOXP2 gene mutation could have been critical for the emergence of proficient language and speech [23].

Enard (2002) showed that the common ancestor of human and mice lived about 70 million years ago. From then until now, the amino acid sequence of the protein encoded by the human FOXP2 gene has produced only three changes, two of which occurred after the human and chimpanzee were separated. This change accelerates the evolution of human language around the time human language emerges [25]. Further researches showed that such gene was positively selected during human evolution. Konopka found that the genetic network that is critical to the language loop has also been positively selected due to the selection pressure imposed on the evolution of the human brain [26]. They believed that the replacement of two amino acids in FOXP2 changes the regulatory pathway of the FOXP2 transcription factor to this gene expression network system. The positive selection of two amino acids in human FOXP2 is a new biological function mechanism of FOXP2 related to language evolution.

The mutation of FOXP2 gene in human evolution is critical for the emergence of proficient language. The mutation and positive selection of FOXP2 gene might have helped human brain adapt for the acquisition of language and speech and the evolution of FOXP2 gene has also been proved to be important to the language related cortical and subcortical brain regions. In addition, current researches suggest that there are many other genes related to human language ability like FOXP2, such as DCDC2, KIAA0319, ATP2C2 and CMIP $[27,28]$. Furthermore, development of the cerebral cortex played an important role in language evolution. ASPM and microcephalin gene are two genes 
that in which mutations will cause severe reductions in the cerebral cortical size of affected humans. Both of them had undergone intense positive selection during human evolution, that is similar to FOXP2 [29,30]. Therefore, the mutation of language-related genes and its further impact on the human brain can be taken as a molecular mechanism of interaction between human language and brain.

\section{A Model for Coevolution of Human Language and Brain}

As stated above, there is a possibility that the development of human language ability and that of brain may be mutually reinforced. Since human language emerged, it had experienced a long period of development and evolved into the modern form with quite high complexity. During such a process, both social conventions and physiological structure have important impacts on language development. Specifically, social conventions provided the constitutional basis for language communications, and physiological structure related with language ability provided the substance basis for speech and logical thinking. In turn, the development of human language can stimulate brain development through more mental exercises. In this section, we will propose a phenomenological model for the coevolution of language and human brain.

\subsection{A Dynamic Model}

For the evolution of language in a given human population, a variable $x_{\mathrm{L}}$ is used to characterize the level of language development, referring to the complexity of language or similar things. We suppose that $x_{\mathrm{L}}$ evolves according to a form of logistic growth with an upper limit [31]. It is reasonable to assume that such an upper limit should be influenced by various factors, especially the level of social development and the developmental level of physiological function that is related to language ability. The level of social development, represented by a parameter $S$, can be supposed to have positive effects on the development of language. For example, the language could be more developed in a society where production activities are well organized. For language-related physiological function, we focus on the average developmental level of brain in the population, such as the development of Broca's area, the size of the human brain, and the descending of the vocal tract, which is represented by a variable $y_{\mathrm{B}}$. For simplification, we set the upper limit of logistic growth as multiplication of the level of social development and the average developmental level of brain. Therefore, the level of language development evolves as follows

$$
\dot{x}_{\mathrm{L}}=\alpha\left(1-\frac{x_{\mathrm{L}}}{\eta y_{\mathrm{B}} S}\right) x_{\mathrm{L}}
$$

Where $\alpha$ is a parameter of growth rate and $\eta$ can be taken as a dimensional parameter.

While the parameter $S$ is set fixed because its dynamics is beyond our discussion, the developmental level of the human brain $y_{\mathrm{B}}$ is supposed to increase during human evolution where a genetic mutation can have played an important role. However, whether a genetic mutation could have evolutionary effects on the developmental level of language depends on whether it could be positively selected under evolutionary pressure. Next, we will model how these evolutionary forces exert their influences on $y_{\mathrm{B}}$.

When a language-related genetic mutation occurs, the population will differentiate into two groups with and without the mutant gene. So we set $y_{0}$ and $y_{1}$ as the developmental level of the human brain for individuals without and with the mutant gene respectively, $n_{0}$ and $n_{1}$ as the sizes of these two groups. So the developmental level of brain in the whole population can be obtained by averaging them as follows

$$
y_{\mathrm{B}}=\frac{1}{n_{0}+n_{1}}\left(n_{0} \cdot y_{0}+n_{1} \cdot y_{1}\right)
$$

The developmental level of the human brain $y_{0}$ and $y_{1}$ can be set to evolve in a form of logistic growth with their own upper limits separately. However, we ignore their dynamics and set them at their steady states, that are the upper limits, on grounds that physiological functions of individuals evolve quickly with their genome given. We set a parameter $K$ to represent the upper limit of the 
physiological level without the mutated gene and $\delta K$ represent the extra increment that can be added to the upper limit of physiological level due to the mutated gene. In this paper, only the case $\delta>0$ is considered, implying a beneficial gene mutation that will promote the function of the human brain. Then, an effect of language development on brain function is introduced, that is, the level of language development in the population will determine the degree to which the extra increment can be gained. Such an effect can be taken as one aspect of the interaction between language and brain. Specifically, a Hill function $f\left(x_{\mathrm{L}}\right)=\frac{x_{\mathrm{L}}^{2}}{L^{2}+x_{\mathrm{L}}^{2}}$ is adopted with the parameter $L$ representing the level of language development where the physiological potential of the gene mutation can be realized in half. So, the developmental level of the human brain is set as follows

$$
\left\{\begin{array}{l}
y_{0}=K \\
y_{1}=K\left(1+\delta f\left(x_{\mathrm{L}}\right)\right)
\end{array}\right.
$$

The evolution of the sizes of the group $n_{0}$ and $n_{1}$ is driven by the competition between individuals without and with the mutant gene. The differential equations can be set by a classical model of survival competition as follows $[32,33]$

$$
\left\{\begin{array}{c}
\dot{n}_{0}=\beta_{0}\left(\phi_{0} y_{0}-n_{0}-\rho n_{1}\right) n_{0}-\gamma_{0} n_{0} \\
\dot{n}_{1}=\beta_{1}\left(\phi_{1} y_{1}-n_{1}-\rho n_{0}\right) n_{1}-\gamma_{1} n_{1}
\end{array}\right.
$$

Where $\beta_{0}, \beta_{1}$ are growth-related parameters for the two groups respectively and $\gamma_{0}, \gamma_{1}$ are parameters of the death rate. The items $\phi_{0} y_{0}$ and $\phi_{1} y_{1}$ represent the available resources for them, supposing that the developmental level of brain in each group will determine the availability of their resources linearly. For example, a higher level of brain development can mean a high capability in resource searching and collecting or an enlargement of resource types that can be utilized. The parameters $\phi_{0}$ and $\phi_{1}$ represent the conversion rate from the developmental level of brain to resource availability. The parameter $\rho$ represents the degree of competition between the two groups with $\rho=1$ representing full competition and $\rho=0$ representing no competition.

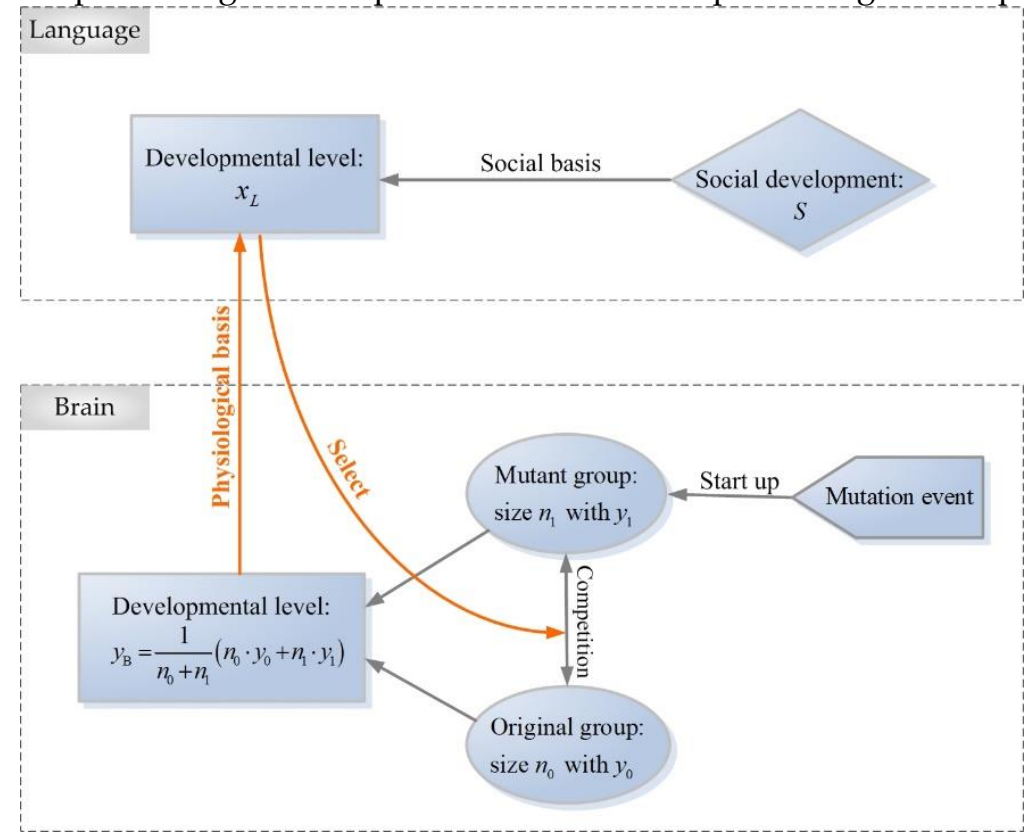

Figure 2. A schematic diagram for the interaction between human language and brain. The effect of brain development on language is shown as "physiological basis", and the effect of language development on brain is shown as "Select".

The interaction between human language and brain can be integrated into a schematic diagram (Figure 2). The developmental level of language can be partly determined by the level of brain development, which is shown as "physiological basis" in the diagram. A language-related gene mutation starts up the differentiation of population into a mutant group with a mutated gene and an original group, who will compete with each other. The developmental level of brain for the population is the average value of the two groups. Whether the mutant group can win over the 
original one, or whether the gene mutation can be positively selected, is influenced by the current level of language development. This effect of language development on brain is shown as "Select" in the diagram.

\subsection{Parameter Analysis and Simulation}

How a mutant language-related gene is selected positively will be analyzed. During the first period, all individuals in the population share the same genome without gene mutation. The developmental level of language approaches to the corresponding steady state $x_{\mathrm{L}} \rightarrow \eta K S$. Then at some time $t_{\mathrm{m}}$, a language-related gene mutates within a given individual and subsequently be transmitted to his/her descendants. Whether such gene mutation can be positively selected and then stimulate the development of language depends on whether the mutant group can win in the survival competition.

In order to focus on the possible results in the survival competition as described in equation (4), the variables $y_{0}$ and $y_{1}$ there are set as constants temporarily. So there are four types of stationary solutions as follows,

$$
\begin{aligned}
& \text { A. } n_{0}=0, n_{1}=0 ; \\
& \text { B. } n_{0}=0, n_{1}=\phi_{1} y_{1}-\gamma_{1} / \beta_{1} ; \\
& \text { C. } n_{0}=\phi_{0} y_{0}-\gamma_{0} / \beta_{0}, n_{1}=0 ; \\
& \text { D. } n_{0}=\left[\phi_{0} y_{0}-\gamma_{0} / \beta_{0}-\rho\left(\phi_{1} y_{1}-\gamma_{1} / \beta_{1}\right)\right] /\left(1-\rho^{2}\right), n_{1}=\left[\phi_{1} y_{1}-\gamma_{1} / \beta_{1}-\rho\left(\phi_{0} y_{0}-\gamma_{0} / \beta_{0}\right)\right] /\left(1-\rho^{2}\right) .
\end{aligned}
$$

The solution $A$ corresponds to a state of species extinction and the solution $D$ represents a state where the two groups can coexist in the long term. In the solution $B$, the mutant group wins over the group without gene mutation, which means that the mutant gene is positively selected. The result in the solution $C$ is opposite to that in $B$. Which type of stationary solution will be realized depends on the set of parameters. To make sure that the dynamic system approach to the stationary solution $B$ where the mutant group is positively selected, the competitive advantage of the mutant group over the other one has to be significant enough.

Now return to the fact that $y_{0}$ and $y_{1}$ are determined according to equation (3). There are two prerequisites of parameters for positive selection of the mutant group as addressed below.

The first prerequisite is that the gene mutation has to have a significant effect on the developmental level of the human brain according to equation (3). That is, the language development at the moment of gene mutation $x_{\mathrm{L}}\left(t_{\mathrm{m}}\right)$ must have reached the level high enough compared to the parameter $L$ so that $f\left(x_{\mathrm{L}}\right) \square 0$. Since $x_{\mathrm{L}}$ is approaching to its steady state $\eta K S$ before the gene mutation occurs as shown in equation (1), it implies that the level of social and physiological development, $S$ and $K$, must be high enough to fix potential functions of such mutant gene in the human brain. Imagine that a gene mutation occurs in a society where the level of social and physiological development is quite low, there is no chance for individuals to exercise new potential skills related to the mutant gene. Even the steady state $\eta K S$ is high enough, but if the current developmental level of language at the moment when the gene mutation occurs is far from its steady state, it is also possible $f\left(x_{L}\right) \rightarrow 0$. In this case, the timing of gene mutation can be crucial.

The second prerequisite involves the extent that the extra increment in brain development due to a gene mutation is transformed into the competitive advantage of the mutant group over the other one in survival competition as shown in equation (4). That is, the available resources $\phi_{1} y_{1}$ have to be more enough compared to $\phi_{0} y_{0}$. As shown in equation (3), this prerequisite requires that the parameter $\delta$, representing the relative amplitude of extra increment in developmental level of brain, has to be large enough so that $y_{1}$ has significant increase compared to $y_{0}$. It also requires that the ratio $\phi_{1} / \phi_{0}$, representing the comparative advantage of conversion rate from the developmental level of brain to resource availability, has to be high enough.

We now show a simulation where a gene mutation is positively selected and subsequently drive language development (Figure 3). First, the level of language development grows steadily toward to its corresponding limit. Suppose a gene mutation occurs at $t=300$ when $n_{1}$ is reset to 1 , 
representing that a language-related gene is mutated in a single individual. The parameters are set to meet prerequisites for the mutant gene to be positively selected. So, $n_{1}$ grows steadily and dominates the whole population in the end (Figure 3a). The winning of individuals with the mutant gene in the survival competition drives the average level of the human brain $y_{\mathrm{B}}$ towards a higher level according to equation (2). As a result, the development of language $x_{\mathrm{L}}$ will approach a higher level (Figure 3b).

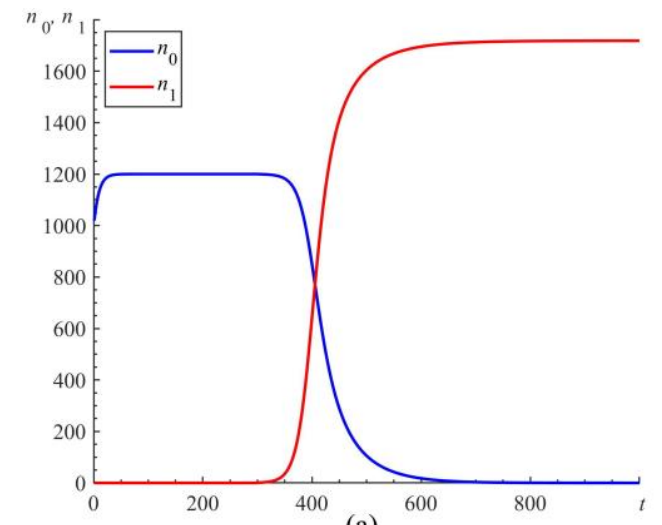

(a)

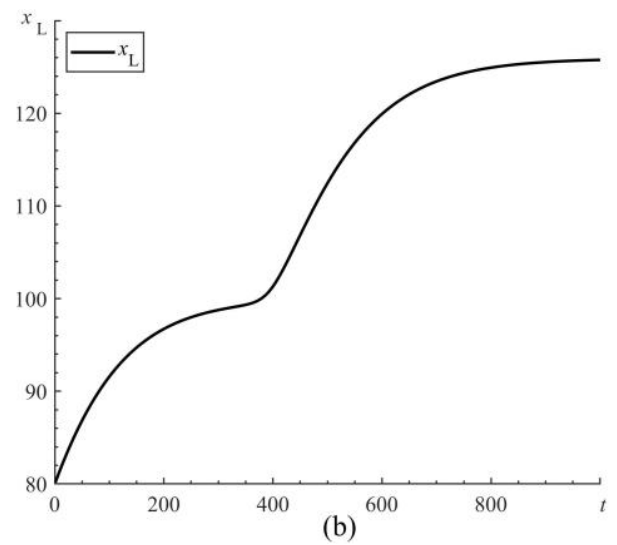

(b)

Figure 3. A simulation for coevolution of human language and brain. The parameters are $\alpha=0.01$, $\eta=1, S=1, K=100, \delta=0.3, L=50, \quad \beta_{0}=0.0001, \quad \beta_{1}=0.0001, \phi_{0}=20, \phi_{1}=20, \rho=0.8$, $\gamma_{0}=0.08, \gamma_{1}=0.08$. The initials are $x_{\mathrm{L}}(0)=80, n_{0}(0)=1000, n_{1}(0)=0$. At the time $t=300$, a gene mutation occurs by resetting $n_{1}=1$. (a) Survival competition between the mutant group and the original one. The gene mutation is positively selected with the mutant group winning over the other one. (b)The evolution of language development. There is a significant increase in the level of language development since a gene mutation occurs.

\section{Discussion}

The properties of complex systems could not be understood without taking the effects of information into account. For many social and biological systems, the learning and adaption of the agents, the interaction among them, and their feedback to the environment are all determined by the information within the system. As one of the major components that compose the world, different from the tight relation between matter and energy, the interaction between information and matter is rarely discussed in previous studies.

In this paper, the interaction of information and matter is addressed. Taking the language and brain as an example, the evidence of their interaction are summarized from the previous studies, and then a dynamic model is suggested.

The model shows that the development of human language and that of the human brain can reinforce each other. That is, the language level can be promoted by advances in brain function due to language-related gene mutations, in turn, whether such mutations can be selected positively depends on the current developmental level of language. The coevolution of human language and brain can be taken as a case of interaction between information and matter.

The brain is crucial for information processing for our human beings. We believe that the interaction of information and matter could be investigated with the progress of brain science. The discussion about the coevolution of language and brain capacity is actually at the macroscopic level. And the model is only a phenomenological one. At the microscopic level, such as the synaptic plasticity, more evidence may be found about the interaction of information and matter.

Acknowledgments: We thank professor Fukang Fang for his helpful discussions and suggestion. Actually, this research topic and related research plan are all initiated by Prof. Fang. Of course, all the possible mistakes and errors in the manuscript are the responsibility of our authors. 
Author Contributions: Z. D. and Y. L. proposed the framework, L. C., J. C. and X. L. developed and analyzed the model, L. C. and Y. L. wrote the paper.

Funding: This research was funded by NSFC, grant number 71731002 and 61573065.

Conflicts of Interest: The authors declare no conflict of interest. The sponsors had no role in the design, execution, interpretation, or writing of the study.

\section{References}

1. Shannon, C.E. A mathematical theory of communication. Bell Syst. Tech. J. 1948, 27, 379-423, $623-656$. Available online: https://doi.org/10.1002/j.1538-7305.1948.tb01338.x

2. Wiener, N. The human use of human beings: Cybernetics and society, 2nd ed.; Houghton Mifflin: Boston, USA, 1954; pp. 17-18.

3. Karlson, P.; Luscher, M. Pheromones: A new term for a class of biologically active substances. Nature 1959, 183, 55-56. Available online: https://doi.org/10.1038/183055a0

4. Binder, J.R.; Frost, J.A.; Hammeke, T.A.; Cox, R.W.; Rao, S.M.; Prieto, T. Human brain language areas identified by functional magnetic resonance imaging. J. Neurosci. 1997, 17, 353-362. Available online: https://doi.org/10.1523/jneurosci.17-01-00353.1997

5. Rijntjes, M.; Musso, M.; Glauche, V.; Buchel, C.; Weiller, C.; Moro, A.; Reichenbach, J. Broca's area and the language instinct. Nat. Neurosci. 2003, 6, 774-781. Available online: https://doi.org/10.1038/nn1077

6. Bogen, J.E.; Bogen, G.M. Wernicke's region - where is it. Ann. N. Y. Acad. Sci. 1976, 280, 834-843. Available online: https://doi.org/10.1111/j.1749-6632.1976.tb25546.x

7. Seghier, M.L. The angular gyrus. Neuroscientist 2012, 19, 43-61. Available online: https://doi.org/10.1177/1073858412440596

8. Oh, A.; Duerden, E.G.; Pang, E.W. The role of the insula in speech and language processing. Brain Lang. 2014, 135, 96-103. Available online: https://doi.org/10.1016/j.bandl.2014.06.003

9. Holloway, R.L. Human paleontological evidence relevant to language behavior. Hum. Neurobiol. 1983, 2, 105-114.

10. Holloway, R.L. Paleoneurological evidence for language origins. Ann. N. Y. Acad. Sci. 1976, 280, 330-348. Available online: https://doi.org/10.1111/j.1749-6632.1976.tb25498.x

11. Sherwood, C.C.; Broadfield, D.C.; Holloway, R.L.; Gannon, P.J.; Hof, P.R. Variability of Broca's area homologue in African great apes: Implications for language evolution. Anat. Rec. 2003, 271A, 276-285. Available online: https://doi.org/10.1002/ar.a.10046

12. Schoenemann, P.T. Hominid brain evolution. In A companion to paleoanthropology, 1st ed.; Begun, D. R., Eds.; Wiley-Blackwell: Chicester, UK, 2013; pp. 136-164.

13. Reader, S.M.; Laland, K.N. Social intelligence, innovation, and enhanced brain size in primates. Proc. Natl. Acad. Sci. USA 2002, 99, 4436-4441. Available online: https://doi.org/10.1073/pnas.062041299

14. Jones, S.; Martin, R.; Pilbeam, D. The Cambridge encyclopedia of human evolution, 1st ed.; Cambridge University Press: New York, USA, 1992; pp. 107-137.

15. Leakey, R. The origins of humankind, 1st ed.; Weidenfeld and Nicolson: London, UK, 1994; pp. 128-135.

16. Laitman J.; Reidenberg J. Advances in understanding the relationship between the skull base and larynx with comments on the origin of speech. Hum. Evol. 1988, 3, 99-109. Available online: https://doi.org/10.1007/bf02436593

17. Lieberman, P.; Crelin, E.S.; Klatt, D.H. Phonetic ability and related anatomy of the newborn and adult human, neanderthal man, and the chimpanzee. Am. Anthropol. 1972, 74, 287-307. Available online: https://doi.org/10.1525/aa.1972.74.3.02a00020

18. Lieberman, P.; Crelin, E.S. On the speech of neanderthal man. Ling. Inq. 1971, 2, 203-222.

19. Lieberman, P. The biology and evolution of language, 1st ed.; Harvard University Press: Cambridge, USA, 1984; pp. 323-324.

20. Aitchison, J. The seeds of speech: Language origin and evolution, 1st ed.; Foreign Language Teaching and Research Press (licensed by Cambridge University Press): Beijing, China, 2002; pp. 59-61.

21. Liegeois, F.; Baldeweg, T.; Connelly, A.; Gadian, D.G.; Mishkin, M.; Vargha-Khadem, F. Language fMRI abnormalities associated with FOXP2 gene mutation. Nat. Neurosci. 2003, 6, 1230-1237. Available online: https://doi.org/10.1038/nn1138

22. Lai, C.; Fisher, S.E.; Hurst, J.A.; Vargha-Khadem, F.; Monaco, A.P. A forkhead-domain gene is mutated in a severe speech and language disorder. Nature 2001, 413, 519-523. Available online: https://doi.org/10.1038/35097076

23. Schreiweis, C.; Bornschein, U.; Burguiere, E.; Kerimoglu, C.; Schreiter, S.; Dannemann, M.; Goyal, S.; Rea, E.; French, C.A.; Puliyadi, R.; Groszer, M.; Fisher, S.E.; Mundry, R.; Winter, C.; Hevers, W.; Paabo, S.; Enard, 
W.; Graybiel, A.M. Humanized Foxp2 accelerates learning by enhancing transitions from declarative to procedural performance. Proc. Natl. Acad. Sci. USA 2014, 111, 14253-14258. Available online: https://doi.org/10.1073/pnas.1414542111

24. Enard, W.; Gehre, S.; Hammerschmidt, K.; Holter, S.M.; Blass, T.; Somel, M.; Bruckner, M.K.; Schreiweis, C.; Winter, C.; Sohr, R.; Becker, L.; Wiebe, V.; Nickel, B.; Giger, T.; Muller, U.; Groszer, M.; Adler, T.; Aguilar, A.; Bolle, I.; Calzada-Wack, J.; Dalke, C.; Ehrhardt, N.; Favor, J.; Fuchs, H.; Gailus-Durner, V.; Hans, W.; Holzlwimmer, G.; Javaheri, A.; Kalaydjiev, S.; Kallnik, M.; Kling, E.; Kunder, S.; Mosbrugger, I.; Naton, B.; Racz, I.; Rathkolb, B.; Rozman, J.; Schrewe, A.; Busch, D.H.; Graw, J.; Ivandic, B.; Klingenspor, M.; Klopstock, T.; Ollert, M.; Quintanilla-Martinez, L.; Schulz, H.; Wolf, E.; Wurst, W.; Zimmer, A.; Fisher, S.E.; Morgenstern, R.; Arendt, T.; Hrabe De Angelis, M.; Fischer, J.; Schwarz, J.; Paabo, S. A humanized version of foxp2 affects Cortico-Basal ganglia circuits in mice. Cell 2009, 137, 961-971. Available online: https://doi.org/10.1016/j.cell.2009.03.041

25. Enard, W.; Przeworski, M.; Fisher, S.E.; Lai, C.; Wiebe, V.; Kitano, T.; Monaco, A.P.; Paabo, S. Molecular evolution of FOXP2, a gene involved in speech and language. Nature 2002, 418, 869-872. Available online: https://doi.org/10.1038/nature01025

26. Konopka, G.; Bomar, J.M.; Winden, K.; Coppola, G.; Jonsson, Z.O.; Gao, F.; Peng, S.; Preuss, T.M.; Wohlschlegel, J.A.; Geschwind, D.H. Human-specific transcriptional regulation of CNS development genes by FOXP2. Nature 2009, 462, 213-217. Available online: https://doi.org/10.1038/nature08549

27. Mozzi, A.; Forni, D.; Clerici, M.; Pozzoli, U.; Mascheretti, S.; Guerini, F.R.; Riva, S.; Bresolin, N.; Cagliani, R.; Sironi, M. The evolutionary history of genes involved in spoken and written language: Beyond FOXP2. Sci. Rep. 2016, 6, 22157. Available online: https://doi.org/10.1038/srep22157

28. Scerri, T.S.; Morris, A.P.; Buckingham, L.; Newbury, D.F.; Miller, L.L.; Monaco, A.P.; Bishop, D.V.M.; Paracchini, S. DCDC2, KIAA0319 and CMIP are associated with Reading-Related traits. Biol. Psychiat. 2011, 70, 237-245. Available online: https://doi.org/10.1016/j.biopsych.2011.02.005

29. Evans, P.D.; Anderson, J.R.; Vallender, E.J.; Gilbert, S.L.; Malcom, C.M.; Dorus, S.; Lahn, B.T. Adaptive evolution of ASPM, a major determinant of cerebral cortical size in humans. Hum. Mol. Genet. 2004, 13, 489-494. Available online: https://doi.org/10.1093/hmg/ddh055

30. Evans, P.D. Reconstructing the evolutionary history of microcephalin, a gene controlling human brain size. Hum. Mol. Genet. 2004, 13, 1139-1145. Available online: https://doi.org/10.1093/hmg/ddh126

31. May, R.M. Simple mathematical models with very complicated dynamics. Nature 1976, 5560, 459-467. Available online: https://doi.org/10.1038/261459a0

32. Hirsch, M. Systems of differential equations which are competitive. Siam J. Math. Anal. 1982, 13, 167-179. Available online: https://doi.org/10.1137/0513013

33. Rescigno, A.; Richardson, I.W. The struggle for life: I. Two species. Bull. Math. Biophys. 1967, 29, 377-388. Available online: https://doi.org/10.1007/bf02476909 